\title{
The role of non-connective discourse cues and their interaction with connectives
}

\author{
Ludivine Crible and Vera Demberg \\ Université catholique de Louvain | Universität des Saarlandes
}

The disambiguation and processing of coherence relations is often investigated with a focus on explicit connectives, such as but or so. Other, non-connective cues from the context also facilitate discourse inferences, although their precise disambiguating role and interaction with connectives have been largely overlooked in the psycholinguistic literature so far. This study reports on two crowdsourcing experiments that test the role of contextual cues (parallelism, antonyms, resultative verbs) in the disambiguation of contrast and consequence relations. We compare the effect of contextual cues in conceptually different relations, and with connectives that differ in their semantic precision. Using offline tasks, our results show that contextual cues significantly help disambiguating contrast and consequence relations in the absence of connectives. However, when connectives are present in the context, the effect of cues only holds if the connective is acceptable in the target relation. Overall, our study suggests that cues are decisive on their own, but only secondary in the presence of connectives. These results call for further investigation of the complex interplay between connective types, contextual cues, relation types and other linguistic and cognitive factors.

Keywords: coherence relations, discourse cues, disambiguation, information density

\section{Introduction}

When reading a text or listening to a speaker, readers and listeners do not process the content of individual sentences as a random juxtaposition of propositions, but instead mentally construct coherence relations between these propositions (Mann \& Thompson 1988). Such pragmatic inferences happening above sentence level include relations of addition, contrast, consequence, temporality or condition, among others. They are often facilitated by the use of explicit connectives, 
such as and, but or so, which function as processing instructions "constrain[ing] the interpretation of the utterances that contain them" (Blakemore 1987: 105). This facilitation has been repeatedly evidenced in comprehension and processing studies (e.g., Millis \& Just 1994; Sanders \& Noordman 2000; van Silfhout et al. 2015).

A substantial amount of discourse research has focused on the role of connectives in coherence relations (Knott \& Dale 1994), comparing "explicit" (with a connective) and "implicit" (without one) contexts across a number of factors, such as the cognitive complexity of the given relation (Hoek et al. 2017) or its expectedness (Asr \& Demberg 2012). However, connectives are but one type of cue that helps readers and listeners retrieve the intended coherence relation. For instance, they can be substituted by "alternative lexicalizations" (Prasad et al. 2008) such as "that is largely due to", which encode the meaning of the relation (causality, in this case). Other cues include focus markers for contrast relations (Carlson 2014), complementizers for consequence (Rohde et al. 2017) or verb tense for temporality (Grisot 2018). Das and Taboada (2018) provide a comprehensive corpus study of discourse cues or "signals" (in their terminology), covering features such as polarity, tense, reference, semantic relations and many others. They showed that, in fact, most coherence relations are signalled by cues other than connectives, and that only $10.65 \%$ of all relations instances in their corpus are exclusively signalled by connectives.

There is thus an extensive body of research on the individual roles of connectives and other discourse cues in the marking and processing of coherence relations. By contrast, the interaction between connectives and other cues has only recently started to be systematically investigated from corpus-based (Crible 2020; Hoek et al. 2019; Das \& Taboada 2019) or experimental methods (Crible \& Pickering 2020; Grisot \& Blochowiak 2019). These studies point to a multifactorial phenomenon of co-occurrence between connectives and other cues, which is impacted by cognitive factors of complexity and information density. The resulting picture remains quite complicated and varies with the coherence relation under scrutiny. More research in this direction is therefore needed to better understand the conditions that favor the co-occurrence between connectives and other cues, and the processing effect of such co-occurrence on discourse comprehension.

In this paper, we investigate the role of non-connective discourse cues in the disambiguation of coherence relations. Our overarching hypothesis is that contextual cues (other than connectives) contribute to inferential processes by making the coherence relation more explicit than a similar version of the relation without such cues. In (1), the contrast between the two clauses is highlighted by the repetition of "plans on $+\mathrm{V}$-ing", whereas there is no such parallel structure in (2), yet the meaning is roughly the same. Using similar manipulations of parallelism, Crible 
and Pickering (2020) showed that items such as (1) are easier to process online than (2). We further argue that such cues also make the contrastive relation easier to disambiguate offline, as they emphasize the comparison between segments.

(1) Jess plans on staying inside. Don plans on taking a walk.

(2) Jess wants to stay inside. Don plans on taking a walk.

We further investigate two factors that potentially qualify the role of discourse cues. First, we compare conceptually different relations, namely consequence (positive causal) and contrast (negative additive). Negative relations such as contrast tend to be reinforced by contextual cues besides the connective more frequently than positive relations such as consequence (Crible 2020; Das \& Taboada 2018). Therefore, we expect to find a stronger effect of cues on the disambiguation of contrast than consequence relations. The latter are arguably equally easy to disambiguate with or without cues, while contrast without cues would be more ambiguous.

We also focus on the degree of explicitness of the discourse relation by manipulating the type of connective that is used: either no connective (implicit relation, see Examples (1) and (2) above), the vague connective and (underspecified relation, Example (3)) or the specific connectives but and so (explicit relation, Example (4)). We expect that discourse cues have a more crucial disambiguating role in implicit and underspecified relations, where less information is available than in explicit relations.

(3) Jess wants to stay inside and Don plans on taking a walk.

(4) Jess wants to stay inside but Don plans on taking a walk.

We report on the results of two experiments using different methodological paradigms (connective insertion and forced choice tasks) in order to pin down the exact contribution of non-connective cues in the disambiguation of coherence relations. The paper is structured as follows. Section 2 reviews previous studies on the processing of coherence relations and their cues, and develops the hypotheses of the present study. Section 3 reports on the method and findings of two experiments. Section 4 discusses the results and limitations, before we conclude in Section 5.

\section{Processing coherence relations and their cues}

The present approach to coherence relations and discourse processing is psycholinguistic and experimental in nature, and the following literature review 
reflects this methodological orientation. However, its motivation and hypotheses are also partly grounded in cognitive corpus linguistics and discourse analysis, so that corpus-based research will be reviewed when relevant.

\subsection{Coherence relations}

Coherence relations are mental representations of the connection between two (or more) utterances, inferred on the basis of linguistic cues and world knowledge. Their meaning is more than the sum of the meanings of each individual utterance (Sanders et al. 1993: 93) and requires an additional step of discourse-level (i.e., above the sentence) processing. Consider the following examples:

(5) The students knew their lesson well. They were confident about their results.

(6) The students knew their lesson well. They were anxious about their results.

(7) The students knew their maths lesson well. They did not study their history lesson.

(8) The students knew their maths lesson well. They were waiting for their results.

The relation between knowing one's lesson and being confident about exam results is one of consequence (5), which could be expressed by so or as a result. By contrast, anxiety is unexpected in this situation, so that the concessive relation in (6) would better be expressed by but or nonetheless. In (7), the difference between the two situations generates a comparison or contrast that can be rendered by but or whereas. The link between the two utterances in (8) is less specific as it merely points at the continuity between events and is probably best represented by the additive conjunction and. These examples show only four types of coherence relations among many more, and illustrate how context-sensitive their disambiguation is.

Relations also differ in how often they are marked explicitly. There is growing converging evidence that shows that not all relations are equal in their degree of explicitness in discourse production (Crible 2020). Studies have related such differences to factors of expectedness (Asr \& Demberg 2012), others to cognitive complexity. Sanders et al.s (1993) Cognitive Approach to Coherence Relations (henceforth CCR) defines relations on the basis of binary dimensions: a relation is either additive or causal, positive or negative, in basic or non-basic order, objective or subjective (see Hoek et al. 2019 for recent further distinctions). Some of 
these characteristics are directly related to cognitive complexity. ${ }^{1}$ In particular, a number of studies have shown that negative relations are cognitively more complex than positive ones: they are acquired later (Evers-Vermeul \& Sanders 2009), induce a processing cost (Sanders \& Noordman 2000) and are more often explicit than their positive equivalents (Hoek et al. 2017). Such differences can reasonably be expected to affect the marking of these relations beyond connectives.

\subsection{Discourse connectives}

Connectives are optional procedural expressions which help "integrate their host utterance into a developing mental model of the discourse in such a way as to make that utterance appear optimally coherent" (Hansen 2006: 25). This theoretical definition, pointing at a facilitating effect of connectives, has been confirmed empirically in studies of processing and comprehension (Degand \& Sanders 2002; Millis \& Just 1994; Traxler et al. 1997; van Silfhout et al. 2015). The role of connectives in coherence relations is thus prominent, and they are considered the prototypical markers for coherence relations. For example, a relation of cause is often marked by because or since, a result by so or as a result and a contrast by but or on the contrary. Connectives are typically coordinating or subordinating conjunctions or adverbials (Prasad et al. 2008).

However, there is no one-to-one mapping between connectives and relations, as some of these expressions are famously polyfunctional. Some connectives are ambiguous: while can be concessive or temporal, since temporal or causal, actually concessive or reformulative, etc. The connective and is particularly notorious in terms of how many relations it can occur in: while most connectives can mark only one or a small number of relations, and is compatible with relations as diverse as consequence, contrast, temporality and concession (Kitis 2000; Pander Maat 1999; Spooren 1997). Therefore, and is regarded as an underspecified connective: it encodes addition but can be pragmatically enriched. For our study, it is particularly relevant to consider the occurrence of and in consequence and contrast relations. Corpus data reveals that and is more frequently found in consequence than contrast relations (Prasad et al. 2008; Crible 2020) - and both relations are much less frequent than the additive relation. This difference in frequency also correlates with results on acceptability judgments: Crible and Demberg (2020) found lower ratings when and was used in contrastive contexts compared to consequence relations.

1. In CCR, cognitive complexity is first based on logic, and then corroborated by evidence from age of acquisition and ease of processing. It therefore relates to the mental representation of a relation and its impact on production and comprehension. 
This discrepancy between the semantic-pragmatic range of different connectives bears consequences on their processing cost. Murray (1997) and Cain and Nash (2011) showed longer reading times for contrast or consequence relations connected by and than by more "appropriate" connectives such as but or so. This effect suggests that weak connectives like and provide incomplete instructions and require the reader or listener to put more effort into the inference process in order to disambiguate the coherence relation. Hence, beyond differences in meaning and use, connectives do not form a homogenous category and differ in how strongly or specifically they signal a coherence relation.

\subsection{Other cues}

Besides connectives, a wide array of linguistic features contribute to making coherence relations explicit. In the Penn Discourse TreeBank 2.0 (henceforth PDTB, Prasad et al. 2008), 624 relations were considered to be marked by an alternative lexicalization (henceforth AltLex), against 18,459 by a connective. AltLexes correspond to phrases which lexically encode the meaning of the relation but are not grammaticalized as connectives (e.g. the most likely reason for this disparity, after that). In the more recent PDTB 3 (Prasad et al. 2018), syntactic constructions specific to a coherence relation (AltLex-C) have been added to the coding scheme, and include structures such as "such NP that" (consequence) or "so [too] <aux> NP" (similarity). In their attempt to always attach a coherence relation to a linguistic element (lexical or syntactic), the PDTB thus adopts an encompassing view of discourse cues.

The same observation has led Das and Taboada (2018) to revisit the "explicit" vs. "implicit" divide regarding coherence relations. In their corpus, a mere $7.26 \%$ of the 21,400 annotated relation tokens are not signalled by connectives or other cues, and another $10 \%$ are exclusively marked by a connective. In their RST Signalling corpus, cues include connectives but also referential chains, lexical fields, semantic relations, syntactic units, graphical signs, and even genre features. These elements are annotated as "signals" for particular relations as soon as they are supportive of the meaning of that relation: a colon may signal an elaboration relation, antonyms can signal a contrast. About a third of their data is signalled by two signals or more (dropping to $\mathbf{1 2 . 2 2} \%$ for relations signalled by three signals or more). In a later paper, Das and Taboada (2019) focus on these signal clusters (including at least one connective) and suggest that additional signals may serve to specify the type of coherence relation (mononuclear or multinuclear) when the connective does not fully specify it itself, or when it is potentially ambiguous between multiple relations, although the co-occurrence between connectives and other signals is not restricted to these two conditions. 
A related approach was recently taken by Crible (2020; in press), who coded a number of lexical, syntactic and semantic features in addition to the connective (i.e., only for relations already marked by an explicit connective) regardless of the conceptual link between these features and the relation. Crible's more systematic approach to discourse cues thus allowed her to distinguish between recurrent "configurations" and predictive "signals", which are quite specific to a given relation. For instance, verb polarity or verb tense are pervasive features that are shared across many relation types (e.g., negation is not restricted to contrastive or concessive relations), whereas hyponymy and parallelism were identified as statistically predictive of the specification and contrast relations, respectively.

Crible (2020) found a robust association between the strength of the connective (i.e., how specific vs. polyfunctional it is) and the presence of such signals: weaker connectives are more likely to co-occur with other signals than more specific connectives. She relates this observation to the Uniform Information Density hypothesis (henceforth UID, Levy \& Jaeger 2007). The latter claims that speakers tend to convey information (as measured by the surprisal of a linguistic unit in context) at a uniform rate, avoiding peaks and troughs in information density. This means that they may tend to omit a signal or choose one with a short encoding, such as and, if the information is already available in the context: if the coherence relation is explicit enough through various cues in the context, a weak connective (or indeed, no connective at all) might suffice. By contrast, Crible found no effect of the cognitive complexity of the coherence relation on the presence or absence of cues, which she explains by the presence of the connective. Experimental validation of these corpus-based results is however necessary to better control and measure the effect of contextual cues in relations with vs. without an explicit connective.

Grisot and Blochowiak (2019) report on a relevant study design for this question. They present a reading study and acceptability judgments for temporal relations signalled both by a connective and by verbal tense, in French texts. The authors find no significant interaction between the two sources of information, suggesting that both tenses are equally acceptable and easy to process with both connectives. Their results also show that the co-occurrence of these cues leads to faster reading times. This is in line with surprisal theory (Hale 2001): reading times are shorter for highly predictable words, and redundant signals are predictable from one another. In our opinion, however, this data does not allow for any conclusions on the validity of the Uniform Information Density hypothesis, contrary to claims in Grisot and Blochowiak (2019). UID only refers to language production but does not make any predictions about language comprehension 
(which is covered by surprisal theory). ${ }^{2}$ Grisot and Blochowiak (2019) conclude that processing temporal relations involves both world knowledge and linguistic knowledge, and that the grammatical (non-)optionality of cues may interfere with their distribution and co-occurrence.

\subsection{Predictions}

Previous studies reviewed above have identified a number of potential factors impacting the presence and effect of discourse cues. In the following experiments, we have retained three dimensions along which a coherence relation is expected to be more or less easy to disambiguate:

- the presence of additional cues;

- the type of coherence relation;

- the presence and strength of a connective.

The first and main hypothesis ( $\left.\mathrm{H}_{1}\right)$ thus tests the disambiguation role of nonconnective cues and compares discourse relations conveying the same ideas with and without such reinforcing cues. We expect cues to facilitate disambiguation, as suggested by Das and Taboada (2019). To test the effects of cue presence, we focus on consequence relations vs. contrastive relations. For consequence relations, we chose to signal the relation via resultative verbs that lexically encode the meaning of consequence, such as to end up or to result, similar to AltLexes in the PDTB (Prasad et al. 2008). Consequence relations without such cues need to be inferred based on world knowledge only and are therefore less explicit than the versions with cues, where the causal inference is lexically encoded in the verb. Examples (9) and (10) illustrate the conditions with and without cues of a consequence item, respectively.

(9) Males have been proven to be more skilled at sports. It allows them to win in mixed competitions.

(10) Males have been proven to be more skilled at sports. They often outperform females.

2. Asr and Demberg (2012) applied the UID hypothesis to a corpus study of explicit and implicit relations. They mention that their assumption strongly connects production patterns with readers' expectations, such that the expectedness of a relation from the reader's perspective might affect the writer's use of connectives, thus linking production with comprehension. Still, the UID hypothesis is used to predict corpus-based frequencies, and the authors do not address its possible effect on processing data, unlike Grisot and Blochowiak (2019). 
Contrast relations in our stimuli are signalled via parallel constructions and/or exact antonyms, as in (11). Contrast relations without cues only involve weak semantic opposition and require more inferencing, as in (12).

\section{(11) The Belgian government decided to create a new tax on solar panels. The French government decided to remove the existing tax. \\ (12) The Belgian government decided to create a new tax on solar panels. Non- renewable energies have been untouched.}

Cues for both consequence and contrast were selected on the basis of Crible's (in press) corpus study of "predictive signals" (cf. Section 2.3). We do not exclude the possibility that more potential cues can be identified in the conditions with cues. However, we made sure that no semantic or syntactic feature of the items in the conditions without cues could be conceptually linked to the notion of contrast.

The second hypothesis $\left(\mathrm{H}_{2}\right)$ concerns the role of cues across different coherence relations. Previous studies suggest that coherence relations are not equal in their typical degree of explicitness. Conceptual differences between relations might be responsible for a weaker or stronger reliance on contextual cues in order to infer the target relation. In our case, we will compare contrast and consequence relations. In Sanders et al's (1993) CCR approach, contrast is categorized as "additive negative", that is, there is no causality component and the two segments negate each other on some aspect; consequence is "causal positive", meaning there is causal inferencing and continuity between the two segments. The literature suggests that the negative relation of contrast is more complex than positive consequence. Therefore, the disambiguation of contrast is expected to be more impacted (i.e., made easier) by the use of cues, where consequence should be equally easy to disambiguate with or without cues.

The third hypothesis $\left(\mathrm{H}_{3}\right)$ targets connectives and compares the underspecified connective and, which can be used in contrast and consequence relations (see Crible \& Demberg 2020; Crible 2020), with the stronger options so for consequence and but for contrast. One of the experiments will also use materials with no explicit connective. Our basic assumption is that strong connectives reflect strong (i.e., clear, unambiguous) relations, so that but and so will be preferred over and when participants are confident about the disambiguation. An alternative hypothesis $\left(\mathrm{H}_{3}{ }^{\prime}\right)$ could be drawn from the Uniform Information Density hypothesis (Levy \& Jaeger 2007). This would suggest that, if the relation is clear enough through contextual cues, weaker connectives will become preferable, as the connective is not needed to signal the relation, and omitting it saves the speaker some production efforts. $\mathrm{H}_{3}$ ' is thus about balancing information sources, whereas $\mathrm{H}_{3}$ relates to ambiguity resolution. 
All three factors investigated in this study and their related hypothesis boil down to the following overarching research question: What makes discourse processing easy or difficult? We will strive to answer it through the particular lens of relation disambiguation and acceptability judgments. More specific hypotheses will be drawn for each of the two experiments, depending on the paradigm at hand.

\section{Experiments}

This study targets the relative ease of offline disambiguation of coherence relations, from multiple methodological paradigms (continuation selection, connective insertion and forced choice task), in order to avoid task-induced biases. We first report the results of a norming study before turning to the two main experiments.

\subsection{Norming study: Continuation selection task}

\subsubsection{Materials and procedure}

We used a continuation selection task as a norming study in order to assess the coherence and naturalness of the materials in the two signalling conditions (with and without cues). This pretest ensures that all materials are equally acceptable, i.e., that the version with cues is not more or less preferred than the version without cues, independently of which connective is used in the sentence. If the materials are carefully constructed, they should not differ in participants' preference.

We constructed pairs of sentences that represent either a contrast or a consequence relation. In each item, the first clause (henceforth $\mathrm{S}_{1}$ ) remains the same across the two signalling conditions: only the second clause ( $\left.\mathrm{S}_{2}\right)$ changes depending on whether cues such as resultative verbs or antonyms are present or not (see Examples 9-12 above). This manipulation does not change the nature of the relation, only its degree of ambiguity or explicitness, using the selected cues presented in Section 2.4. For each S1, the continuation options include the S2S with and without cues, as well as a third unrelated and non-sensical option taken from other materials used in pilot trials. An example item with the three options is given in (13).

(13) The southern states share the same view on environment and/but...

A. consensus on foreign policy is hard to find. (contrast, without cues)

B. gay people live in the open. (non-sensical)

C. the northern states have another. (contrast, with cues) 
In this example, there is a contrastive relation in conditions $\mathrm{A}$ (without cues) and $\mathrm{C}$ (with cues), although in the latter, the contrast is much more direct with the antonyms southern and northern, whereas in the former, the relation between environment and foreign policy is only one of category membership (they are different topics of political debate). Although we acknowledge that semantic manipulations are not trivial, we believe that the usage of clear lexical cues (antonyms for contrast, resultative verbs for consequence) allowed us to operationalize our experimental conditions. The "with cues" and "without cues" conditions are meant to be different by design, and these differences make it necessary to norm the stimuli, to make sure that they do not significantly differ in acceptability.

In total, the materials contained sixteen contrast and consequence relations (eight different Sis for each relation type). All Sis ended with a connective: half of them with and, the other half with but or so (depending on the coherence relation). In addition, we included four causal and four temporal relations that acted as filler items. The connective for filler items was either because or as. We distributed these items in two lists of twenty-four trials so that participants would never see the same $S_{1}$ twice. They also never saw the same continuation option twice. Table 1 summarizes the experimental conditions of the norming study.

Table 1. The $2 \times 2$ experimental design of the continuation selection task

\begin{tabular}{llc}
\hline Relation & Connective & No. of items \\
\hline consequence & and & 4 \\
consequence & so & 4 \\
contrast & and & 4 \\
contrast & but & 4 \\
\hline
\end{tabular}

Participants had to read the beginning of a sentence containing the first clause and the connective. They then had to select among the three possible continuations by clicking on their preferred option. The order of presentation of the trials and of the continuations (with and without cues, non-sensical) was randomized for each participant.

This norming study was programmed on LingoTurk (Pusse et al. 2016) and hosted on Prolific. All studies in this paper were carried out in accordance with the recommendations of the American Psychological Association, with written informed consent from all subjects. The protocol was approved by the ethics committee of the Deutsche Gesellschaft für Sprache (DGfS). There was no time constraint, and each participant completed the task in about 7 minutes on average. 


\subsubsection{Participants}

Two groups of 35 participants ( $57.33 \%$ females) were recruited on the crowdsourcing platform Prolific (www.prolific.co) and remunerated for their work $(\mathfrak{E} 1.5)$. They had to be adults and native speakers of English. The two groups each saw one of the two lists of materials.

\subsubsection{Results}

We excluded from our analysis cases where the participants selected the nonsensical continuation ( $3 \%$ of the data) and the filler items. We ran a logistic mixed-effect regression model on the selection of continuations with or without cues, with participants and items as random effects: using forward model selection with anova (Baayen et al. 2008), adding Connective (and vs. but/so) as factor did not improve the baseline model $\left(\Delta \chi^{2}=0.3581, \Delta d f=1, p>.05\right)$, nor did adding Relation $\left(\Delta \chi^{2}=0.1515, \Delta d f=1, p>.05\right)$. In other words, we found no main effect of connective type nor of relation on the selection of continuations with or without cues. The distribution is particularly smooth in consequence trials, as can be seen in Figure 1. In contrast relations, there is a slight but non-significant preference for continuations with reinforcing cues when the connective is and but not when it is but.

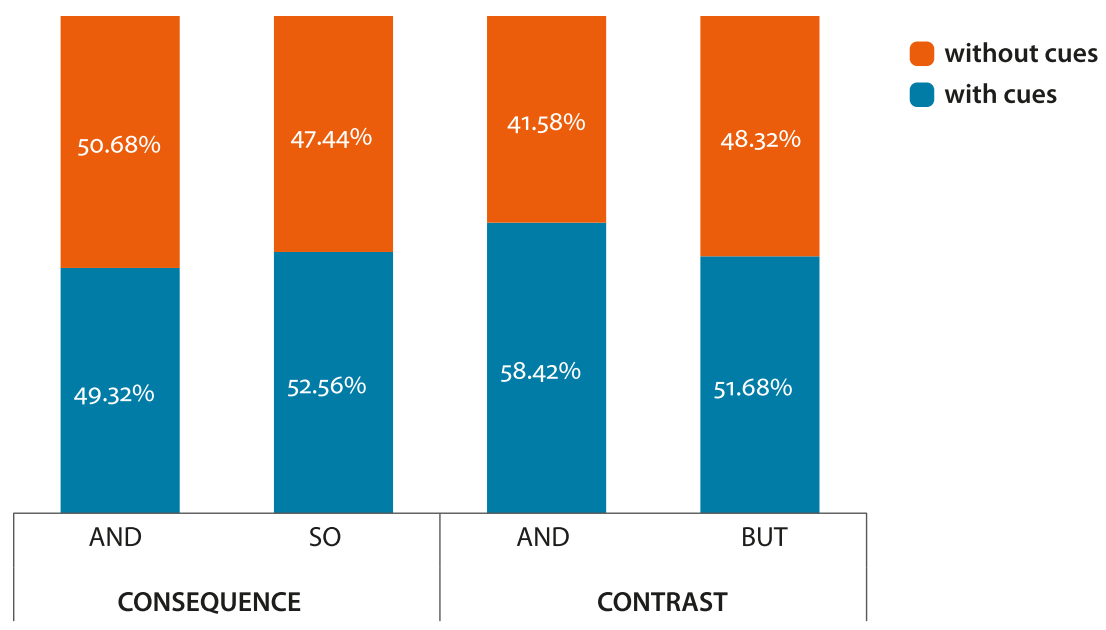

Figure 1. Proportions of the "with" and "without cues" continuations across conditions

\subsubsection{Discussion}

The non-significant difference between all conditions suggests that the materials with and without cues do not differ in acceptability when presented in a continuation selection task. Both continuation types (with and without cues) are equally 
valid and natural to participants for the same given $S_{1}$, and this holds independently of whether the relation is marked with a weak connective and or a more specific connective so or but. This result thus enables us to use these materials in other tasks, knowing that all versions are equally acceptable.

\subsection{Experiment 1: Connective insertion task}

In order to measure the effect of non-connective cues on the disambiguation of "implicit" (i.e., not marked by a connective) coherence relations, we conducted a connective insertion task. This experiment allows us to identify the specific contribution of discourse cues on relation disambiguation independently from the instructions of connectives. In this respect, we expect cues such as resultative verbs for consequence or antonyms for contrast to make the coherence relation more explicit and thus easier to disambiguate $\left(\mathrm{H}_{1}\right)$. In our task, this ease of disambiguation will be reflected by the consensus or percentage of agreement between participants regarding the connective they would insert to connect the two arguments (clauses) of the relation. We further expect that cues will be more beneficial in contrast relations than consequence relations due to their conceptual differences $\left(\mathrm{H}_{2}\right)$. In other words, the difference between agreement rates with and without cues should be larger in contrast than in consequence.

\subsubsection{Materials and procedure}

We used the materials from the norming study, for the same total number of sixteen items plus eight fillers. In one list, half the materials (four contrast and four consequence) were presented in the "with cues" condition, and the same materials were "without cues" in the second list (Table 2).

Table 2. The $2 \times 2$ experimental design of the connective insertion task

\begin{tabular}{llc}
\hline Relation & Cues & No. of items \\
\hline consequence & with & 4 \\
consequence & without & 4 \\
contrast & with & 4 \\
contrast & without & 4 \\
\hline
\end{tabular}

In each trial, the pair of sentences $\left(S_{1}+S_{2}\right)$ was presented on the screen without any connective but with an empty box between the two clauses, where the connective should be. The first clause always ended with a double forward slash "//" and the participants were instructed that they could interpret this symbol as 
any punctuation sign they want (comma, full stop, etc.). Participants had to dragand-drop the connective that best fit the context according to them, from a list of options including two consequence (so, as a result), two contrast (by contrast, whereas), one causal (because), one temporal (when) and two conditional connectives (unless, if). The exact instructions were: "For this experiment, you will be asked to read passages of text and indicate the logical link between the two elements of the passages. [...] Please choose the linking phrase that best reflects the meaning of the connection between the sentences." The order of presentation of the options was fully randomized. Participants also had the possibility to enter a manual answer if they considered that none of the proposed options fitted. The experiment was programmed on LingoTurk (Pusse et al. 2016). There was no time constraint for the task, and each participant completed the experiment in about 7 minutes on average.

\subsubsection{Participants}

Two groups of 32 adult native English speakers (70.3\% females) were recruited through Prolific and remunerated (£ 1.5). The data from one participant who entered random and inconsistent answers were removed entirely.

\subsubsection{Results}

All connective insertions were coded as correct or incorrect depending on the adequacy between the semantics of the target relation (contrast or consequence) and that of the inserted connective. For instance, conditional connectives such as if were considered incorrect in the case of a consequence relation, where so or as a result were expected. However, we decided to consider concessive connectives (e.g., although, yet, $n=8$ ) as correct in the case of a contrast relation, because of the conceptual proximity of the two relations (Zufferey \& Degand 2013) and on the basis of previous studies showing the possible use of connectives such as although in contrastive contexts (Asr \& Demberg 2016). Furthermore, we coded causal insertions as correct in consequence relations (because, $n=71$ ), given that cause and consequence are both defined as causal relations in the CCR, with only a difference in the order of events (cause-effect vs. effect-cause). Cause and consequence are considered as subtypes of the same relation in most frameworks, such as the PDTB 2.0 ("reason" vs. "result", Prasad et al. 2008) or Rhetorical Structure Theory (Mann \& Thompson 1988). For several of our items, both causal and consequence interpretations were possible, as in (14).

(14) Air travel used to be very expensive // it was mainly used for business.

Overall, $82.5 \%$ of connective insertions were correct, in a similar proportion across the two target relations ( $85.6 \%$ for consequence, $79.4 \%$ for contrast), as 
shown in Figure 2. We ran a mixed-effect generalized logistic regression model on the data (excluding filler trials), with correct vs. wrong connectives as the dependent variable and participants as random effects. Adding presence vs. absence of cues as factor improved the baseline model $\left(\Delta \chi^{2}=52.872, \Delta d f=1, p<.001\right)$. Adding relation type further improved the model $\left(\Delta \chi^{2}=19.525, \Delta d f=2, p<.001\right)$. The final model with cues and relation as independent variables returned a main effect of cues $(\beta=0.6418, S E=0.2722, p<.05)$ and a highly significant interaction between cues and relations $(\beta=1.3417, S E=0.3944, p<.001)$. This means that, overall, the presence of clear textual cues leads to a higher rate of insertions that match the meaning of the relation, and that, as predicted, contrastive relations without cues were substantially more difficult to disambiguate.

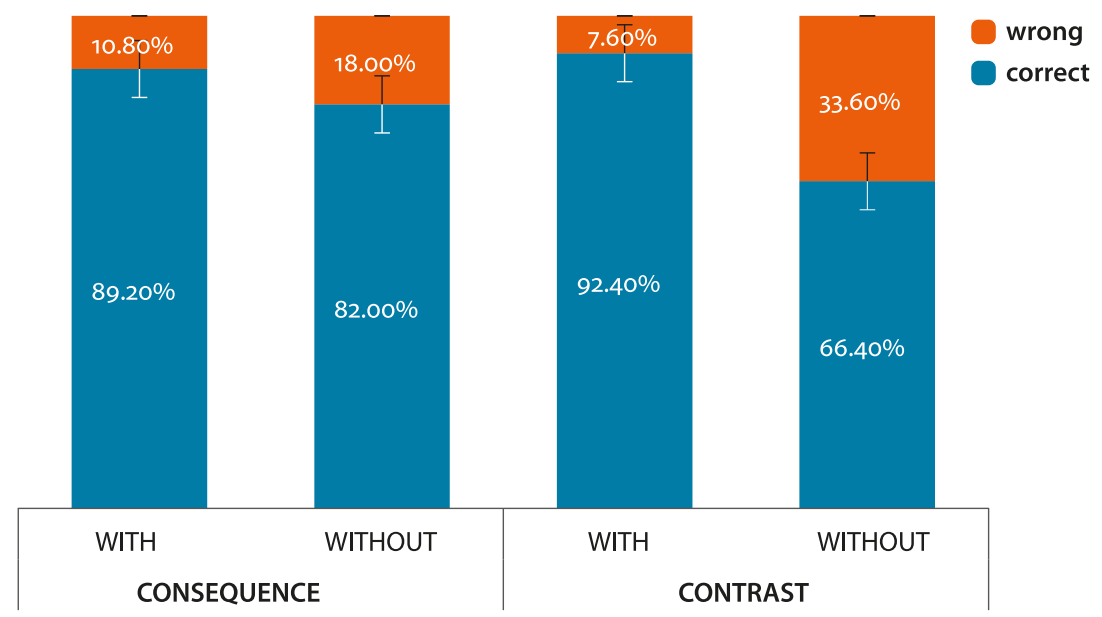

Figure 2. Correct and incorrect connective insertions across conditions

\subsubsection{Discussion}

The connective insertion task confirms our hypothesis $\left(\mathrm{H}_{1}\right)$ on the positive disambiguating role of cues on relation disambiguation, in the absence of explicit connectives. Using materials that have been controlled for their acceptability in the "with" and "without" cues conditions (see the norming study above), we found that the presence of cues is a significant factor in the correctness of the connectives inserted by the participants. This suggests that, given the same first clause $\left(\mathrm{S}_{1}\right)$, a coherence relation is less ambiguous and more easily disambiguated when the S2 contains clear linguistic features associated with the conceptual meaning of the relation (antonyms and parallelism for contrast, resultative verbs for consequence). While the coherence relation can still be disambiguated without such cues in some cases, the agreement level between participants is overall lower without cues ( $74.2 \%$ vs. $90.8 \%)$. 
Furthermore, the regression model showed a highly significant interaction between relations and presence of cues, which indicates that discourse cues have a larger disambiguating role in contrast relations than in consequence relations, thus confirming our second hypothesis $\left(\mathrm{H}_{2}\right)$. Note that there was no significant main effect of relation, which indicates that the contrast and consequence items do not differ overall in terms of their ease of disambiguation. What the interaction instead shows is a higher sensitivity of participants to the presence of cues in contrastive relations, whereas relation interpretation in consequence relations is less impacted by discourse cues. While this result may mitigate the claim that contrast is a more cognitively complex relation than consequence, as suggested by its negative polarity (see CCR), the data clearly support the view that not all relations are equal in their association with discourse cues. In fact, this could indicate that some coherence relations (like contrast) are more linguistically constrained, more sensitive to textual cues than others (like consequence), which would be more based on world knowledge (see the multiple sources of information discussed in Grisot \& Blochowiak 2019).

\subsection{Experiment 2: Forced choice task}

This experiment uses a forced choice task in order to verify that relation disambiguation is indeed easier and more consensual with cues than without, when such cues co-occur with different connectives. We compare the default connectives but and so to the weaker conjunction and, in order to record the level of confidence of participants in their discourse interpretation. We take preferences for and as a sign of low explicitness of the relation and therefore of low confidence of the participants: if participants select the and-version as opposed to but or so, it indicates that they do not commit to a specific coherence relation such as contrast or consequence but prefer to leave the relation unspecified. If, on the other hand, participants prefer but/so over and, it means that they have identified the coherence relation more precisely. In this perspective, we expect to find more selections of but and so with cues, when the relation is arguably clear, and more selections of and without cues, when the relation is more ambiguous and unspecified $\left(\mathrm{H}_{3}\right)$.

An alternative account of connective choices is offered by the Uniform Information Density hypothesis ( $\left.\mathrm{H}_{3}{ }^{\prime}\right)$, whereby ambiguous and can be expected to be preferred in relations with additional reinforcing cues, while so and but should be preferred in relations without cues. Such a behaviour in the participants would reflect a strategy of balancing information sources so as not to overload the marking of the coherence relation: if cues such as antonyms are used in the context, then the relation is sufficiently explicit already and does not require a strong connective to be further marked; on the other hand, if the relation is not made clear 
by the presence of cues, then a stronger connective such as but would prevail, to compensate for the ambiguity of the unsignalled relation. This experiment thus complements the findings of the connective insertion task by adding connectives and their interaction with cues into the picture of relation disambiguation.

\subsubsection{Materials and procedures}

We used the same 24 stimuli ( 16 critical + eight fillers) again for this study. In each trial, participants saw two versions of the same segments next to each other on the screen. The only difference between the two versions was the connective (and vs. but or so). Half of all stimuli contained cues, the other half did not, in equal proportion across relations (Table 3 ). Materials were split into two lists and participants only saw one version of each item (either with or without cues).

Table 3. The $2 \times 2$ experimental design of the forced choice task

\begin{tabular}{lllc}
\hline Relation & Cues & Connective & No. of items \\
\hline consequence & with & and vs. so & 4 \\
consequence & without & and vs. so & 4 \\
contrast & with & and vs. but & 4 \\
contrast & without & and vs. but & 4 \\
\hline
\end{tabular}

Participants were asked to tick a box underneath the version they prefer. The exact instructions were: "Your task will be to read the two versions of the passage and choose the one with the linking phrase that you think fits best in the passage, the one that you would most likely have used in a similar context." The experiment was programmed on LingoTurk (Pusse et al. 2016). There was no time constraint for the task, and each participant completed the experiment in about 8 minutes on average.

\subsubsection{Participants}

Two groups of 35 participants were recruited on Prolific and remunerated $(£ 1.5$ per participant). All participants were adult native English speakers, $64.56 \%$ were female.

\subsubsection{Results}

We report results for critical trials, excluding fillers. Overall, relations connected by but and so were more frequently preferred than versions with and $(71.45 \% \mathrm{vs}$. $28.55 \%)$. However, Figure 3 shows that consequence relations without cues lead to a relatively high rate of preferences for and-versions compared to the other three conditions. 


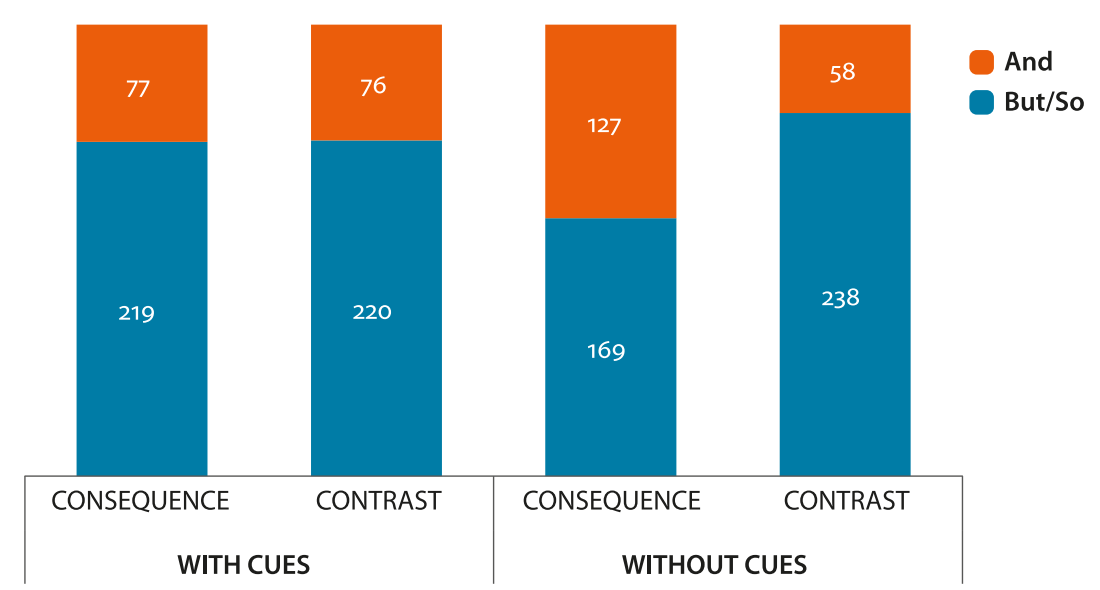

Figure 3. Proportions of preferences for and vs. but or so

We ran a mixed-effects logistic regression model with type of connective (and vs. but/so) as the dependent variable. Adding presence of cues as a factor to a baseline model with participants and items as random effects improved the fit of the model $\left(\Delta \chi^{2}=4.855, \Delta d f=1, p<.05\right)$, so did adding relation type $\left(\Delta \chi^{2}=23.422, \Delta d f=2, p<.001\right)$. The final model returns a main effect of cues $(\beta=0.18544, S E=0.18544, p<.001)$ and a significant interaction between cues and relations $(\beta=-1.22046, S E=0.27984, p<.001)$. These findings indicate that relations with cues lead to a preference for the specific connectives but and so. There is no main effect of relation type (i.e., both relations equally disprefer and overall). However, and is less dispreferred in unsignalled consequence than in unsignalled contrast.

\subsubsection{Discussion}

The forced choice task confirms $\mathrm{H}_{3}$ regarding the use of connectives as indicators of (un)certainty in relation disambiguations. Participants indeed preferred the unambiguous connectives (but, so) more when the relation was reinforced by other cues than when the relation was less explicit. This indicates that, without cues, participants are less confident about the nature of the relation and will more often choose a neutral additive connective, without committing to a more specific interpretation. This result could therefore suggest a "good-enough" strategy by participants in order to avoid mistakes: in experimental settings, participants might feel like there is a "good" answer; choosing and could be a safe choice in this respect. As a result, we can reject the alternative UID account $\left(\mathrm{H}_{3}{ }^{\prime}\right)$, which suggested that weak connectives would be preferred when other strong cues were already in use. 
The above effect mainly holds for the consequence relation. The significant interaction between relations and cues indeed indicates an increased preference for and-versions in unsignalled consequence, whereas and is equally dispreferred in contrast with and without cues. This result suggests that discourse cues make consequence relations more explicit (hence more selections of $s o$-versions). As for contrastive relations, the effect of cues seems to be overridden by an underlying dispreference for and in contrast, as already pointed out by Crible and Demberg (2020), where we found that acceptability ratings were much lower for and in contrast than in consequence, which also echoes the lower frequency of contrastive and in corpus data.

The positive disambiguating role of cues thus appears to be mostly valid for consequence items in this experiment (see the interaction discussed above) but does not seem to apply to contrastive relations as much. The latter appears as quite stable and unaffected by cues, due to the low acceptability of and in contrast regardless of other discourse cues. Comparisons with the previous experiment, as well as further theoretical implications, will be discussed in the General discussion below.

\section{General discussion}

After having established the overall similarity between two versions of the same coherence relation, one where this relation is explicitly reinforced by cues and one where it is not, we were able to show through a connective insertion task and a forced choice task that the presence of non-connective cues indeed helps participants identify the coherence relation holding between two sentences. Our main hypothesis on the disambiguating role of discourse cues $\left(\mathrm{H}_{1}\right)$ is thus confirmed and complements recent (mostly corpus-based) studies investigating such linguistic devices (e.g., Das \& Taboada 2019; Hoek et al. 2019). This result has important implications for psycholinguistic, computational and even acquisitional perspectives, and shifts the attention away from connectives, which had so far been the focus of discourse studies.

We further compared the ease of disambiguation for two coherence relations, namely contrast and consequence, which differ in their conceptual features and associated cognitive complexity: negative relations such as contrast are considered more complex than positive ones like consequence, as reflected in findings from translation (Hoek et al. 2017) or acquisition (Evers-Vermeul \& Sanders 2009). Three conclusions can be drawn from our data in this respect: 
- Contrast is not more complex to disambiguate offline than consequence overall (contrary to what its negative feature might suggest);

- The disambiguation of contrast is, however, more impacted by contextual cues than the disambiguation of consequence relations, as expected $\left(\mathrm{H}_{2}\right)$;

- Finally, the role of cues in contrastive relations disappears when connectives are used, as a result of underlying frequency associations.

It is thus a complex picture of interacting factors that emerges from our data: sensitivity to cues (higher in contrast) and compatibility with specific connectives seem to play a more prevalent role on relation disambiguation, rather than cognitive complexity on its own.

Our third hypothesis targeted the use of connectives either as signs of certainty $\left(\mathrm{H}_{3}\right)$ or as a way to balance information density $\left(\mathrm{H}_{3}{ }^{\prime}\right)$. In consequence relations, where discourse cues were found to have an effect on the choice of connective, it is the first account $\left(\mathrm{H}_{3}\right)$ that seems to be confirmed: and-versions were preferred over so more often in contexts without cues than with cues. We related this finding to a "good-enough" strategy for participants, who do not commit to specific interpretations in the absence of contextual cues. The Uniform Information Density account ( $\mathrm{H}_{3}$, Levy \& Jaeger 2007) is thus not supported by our data. According to UID, the information provided by cues should have been counterbalanced by the selection of the weak connective and instead of the more informative so and but in order not to be redundant and create an information overload. However, in our data, strong connectives were preferred in strong relations (i.e., with cues). Grisot and Blochowiak (2019) also found that speakers read faster and found more acceptable coherence relations (temporal, in their case) where a specific connective is combined with another cue. The authors explain this result by the different nature of the multiple cues combined and suggest that redundancy would only apply to multiple cues of the same nature.

However, the hypothesis by Levy and Jaeger (2007) originally accounted for such clusters of different information sources, including optional and nonoptional ones at the syntactic level. Therefore, we propose that the absence of a UID effect in our data (see $\mathrm{H}_{3}$ ) is rather due to the different language task that is originally targeted by the hypothesis: UID accounts for natural language production, where speakers know what they want to say and have to choose from multiple encoding options. By contrast, the present experiments try to mimic natural production but may nevertheless involve different mechanisms. Our data therefore may reflect reasoning about alternatives and participants' attempt to give a correct answer, rather than spontaneous production. In other words, UID may not account for the results obtained here due to task differences. 
Furthermore, our data could also be biased by the experimental setting: unlike in natural language use, where speakers and writers have many options and where no immediate judgment is involved, participants in an experiment tend to be under the impression that there is a "correct" and a "wrong" answer (this effect is sometimes called a "response bias"). Although our test instructions did not mention a unique right answer but rather pointed at personal preference ("select the connective that you would have used"), we cannot be sure that participants did not interpret them as suggesting that some connective uses are unacceptable. As a result, and could be perceived as the wrong answer based on the participants' assumption that it is too vague, which could explain the low rate of and-selections in the forced choice task. This bias also partly refers to our hypothesis on the participants' certainty and confidence in their disambiguation: once they have identified the target relation, they will pick the connective that best matches that relation (but or so), instead of the more neutral and, if they feel that this is what was expected of them, to measure if they performed the task correctly. Natural language, as can be observed in corpora, and linguistic experiments are inherently different. While it was beyond the scope of this study to address this gap, it should be borne in mind as another factor which might explain our data.

Our two experiments show potentially discordant results regarding the disambiguation of contrast. The connective insertion task first showed that, in the absence of connectives, contrast benefits from cues more than consequence in terms of disambiguation. This differs from the findings of the forced choice task, where it appeared that but was preferred over and regardless of the presence of cues, while discourse cues made consequence relations more explicit and significantly favored the selection of so over and. We explained this difference between relations by the respective frequency of use of and in consequence and contrast, and its respective acceptability in these relations (lower in the latter). By contrast, and easily accommodates causal inferences, as already pointed out in previous theoretical (Luscher \& Moeschler 1990) and corpus-based studies (Spooren 1997). Therefore, the effect of cues on connective choice seems modulated by an a priori compatibility between the connective and the target relation, which is not the case for contrast.

The use of connectives in Experiment 2 thus seems to account for the differences with Experiment 1. Discourse cues indeed appear less crucial when a connective is already present in the context (at least for the contrastive items), in which case it is the acceptability of the connective in the target relation that prevails. We could conclude from this nuanced observation that discourse cues indeed help disambiguating coherence relations, but in a secondary role compared to connectives, which remain the main signal for relations. Discourse cues may be more frequent than actual connectives (Das \& Taboada 2018), but their 
pervasiveness in different contexts and different relations makes their contribution only secondary in the presence of connectives. This finding echoes the types of interactions between connectives and other cues distinguished by Hoek et al. (2019) in a translation study. The authors identified three ways in which cues combine with connectives depending on their frequency of co-occurrence and semantic congruence: "division of labor" (redundant), "agreement" (frequent and congruent), "general collocation" (frequent and incongruent). They conclude that "not all non-connective signals for coherence relations are created equal" (2019:27) and that their investigation requires combining theoretical, corpusbased and experimental methods.

Overall, this study leads us to suggest that a wide array of semantic and cognitive considerations interact in the marking of coherence relations, and that univariate approaches to a single factor are bound to overlook important restrictions brought about by other linguistic dimensions. Through the present experiments, we only investigated three of these dimensions: the presence of textual cues conceptually related to specific relations, the type of coherence relation, the type of connective and its degree of semantic precision. Further research would benefit from including more dimensions, such as discourse genre or text type, as well as replicating our results on other coherence relations and/or with other types of cues.

Finally, we would like to acknowledge limitations to our experiments, which prevent full generalization of our results. Firstly, our sample size is relatively small, especially given recent standards inherited from the growing influence of psychology on (psycho)linguistics (e.g., Brysbaert \& Stevens 2018). Experimental materials designed for discourse studies are particularly challenging to create since they involve the manipulation of semantic features. In particular, items in the unsignalled condition had to make the relation identifiable without any connective or any other clear textual cue. We also did not control for length or word frequency of the materials, given that we were not trying to access online measures of processing but offline disambiguation. An obvious avenue of research is therefore to turn to other methods which allow to capture online processes, such as selfpaced reading or eye-tracking studies, with more controlled (and more numerous) experimental stimuli (e.g., Crible \& Pickering 2020). Lastly, the choice of the linguistic cues to be manipulated was based on a corpus study (Crible in press), but their nature was quite different for the two coherence relations, and one could argue that the cues for contrast (antonyms) were inherently clearer than those for consequence (resultative verbs). Specific effects of different types of cues for a given relation could help distinguishing between their respective disambiguating power. 


\section{Conclusion}

The offline experiments discussed in this paper shed new light on the disambiguating role of non-connective cues for coherence relations. In particular, we tested the effect of antonyms and resultative verbs on the disambiguation of contrast and consequence relations, respectively. We showed that such nonconnective cues indeed help our participants disambiguate the target relation, and that they preferred semantically specific connectives to indicate their confidence in their interpretation. By moving away from the focus on single explicit connectives, and by studying their interaction with other cues instead, we have showed that cognitive and psycholinguistic approaches to discourse can benefit from expanding the range of coherence markers at play, and should also consider multiple factors and their interaction.

This study addresses a number of central issues in pragmatics, cognitive linguistics and psycholinguistics, such as the contribution of specific linguistic elements of different natures to the construal of coherence relations, the role of the Uniform Information Density hypothesis (Levy \& Jaeger 2007) in discourse production, or the difference between production and comprehension. As such, our results open up more research questions and call for replication with other relation types and/or cue types. Only highly controlled experiments teasing apart the processing effects of particular linguistic (and non-linguistic) information sources will allow us to access the full picture of discourse production and comprehension. Considering the role of world knowledge and the high variation that arises at discourse level, this will by no means be a trivial endeavour.

\section{Funding}

Funded by the Deutsche Forschungsgemeinschaft (DFG, German Research Foundation) Project-ID 232722074 - SFB 1102.

\section{References}

Asr, Fatemeh \& Vera Demberg. 2012. Measuring the strength of linguistic cues for discourse relations. In Eva Hajičová, Lucie Poláková, \& Jiří Mírovský (eds.), Proceedings of the COLING workshop on advances in discourse analysis and its computational aspects (ADACA), 33-42. Mumbai, India: The COLING 2012 Organizing Committee.

Asr, Fatemeh \& Vera Demberg. 2016. But vs. although under the microscope. Poster at CogSci 2016, Philadelphia, USA. 
Baayen, Harald, Douglas J. Davidson, \& Douglas M. Bates. 2008. Mixed-effects modeling with crossed random effects for subjects and items. Journal of Memory and Language 59. 390-412. https://doi.org/10.1016/j.jml.2007.12.005

Blakemore, Diane. 1987. Semantic constraints on relevance. London: Basil Blackwell.

Brysbaert, Marc \& Michael Stevens. 2018. Power analysis and effect size in mixed effects models: A tutorial. Journal of Cognition 1(1). 9. 1-20. https://doi.org/10.5334/joc.10

Cain, Kate \& Hannah Nash. 2011. The influence of connective on young readers' processing and comprehension of text. Journal of Educational Psychology 103(2). 429-441. https://doi.org/10.1037/aoo22824

Carlson, Katy. 2014. Predicting contrasts in sentences with and without focus marking. Lingua 150. 78-91. https://doi.org/10.1016/j.lingua.2014.07.008

Crible, Ludivine. In press. The syntax and semantics of coherence relations: From relative configurations to predictive signals. International Journal of Corpus Linguistics.

Crible, Ludivine. 2020. Weak and strong discourse markers in speech, chat and writing: How signals compensate for ambiguity in explicit relations. Discourse Processes 57(9). 793-807. https://doi.org/10.1080/0163853X.2020.1786778

Crible, Ludivine \& Vera Demberg. 2020. When do we leave discourse relations underspecified? The effect of formality and relation type. Discours 26 . https://doi.org/10.4000/discours.10848

Crible, Ludivine \& Martin J. Pickering. 2020. Compensating for processing difficulty in discourse: Effect of parallelism in contrastive relations. Discourse Processes 57(10). 862-879. https://doi.org/10.1080/0163853X.2020.1813493

Das, Debopam \& Maite Taboada. 2018. Signalling of coherence relations in discourse, beyond discourse markers. Discourse Processes 55(8). 743-770. https://doi.org/10.1080/0163853X.2017.1379327

Das, Debopam \& Maite Taboada. 2019. Multiple signals of coherence relations. Discours 26. https://doi.org/10.4000/discours.10032

Degand, Liesbeth \& Ted J.M. Sanders. 2002. The impact of relational markers on expository text comprehension in L1 and L2. Reading and Writing: An Interdisciplinary Journal 15. 739-757. https://doi.org/10.1023/A:1020932715838

Evers-Vermeul, Jacqueline \& Ted J.M. Sanders. 2009. The emergence of Dutch connectives: How cumulative cognitive complexity explains the order of acquisition. Journal of Child Language 36. 829-854. https://doi.org/10.1017/S0305000908009227

Grisot, Cristina. 2018. Cohesion, coherence and temporal reference from an experimental corpus pragmatics perspective. Cham: Springer. https://doi.org/10.1007/978-3-319-96752-3

Grisot, Cristina \& Joanna Blochowiak. 2019. Temporal connectives and verbal tenses as processing instructions. Pragmatics and Cognition 24(3). 404-440. https://doi.org/10.1075/pc.17009.gri

Hale, John. 2001. A probabilistic early parser as a psycholinguistic model. In Proceedings of the Second Meeting of the North American Chapter of the Association for Computational Linguistics on Language Technologies, 1-8. Pittsburgh, PA: Association for Computational Linguistics.

Hansen, Maj-Britt M. 2006. A dynamic polysemy approach to the lexical semantics of discourse markers (with an exemplary analysis of French toujours). In Kerstin Fischer (ed.), Approaches to discourse particles, 21-41. Amsterdam: Elsevier. 
Hoek, Jet, Sandrine Zufferey, Jacqueline Evers-Vermeul \& Ted J. M. Sanders. 2017. Cognitive complexity and the linguistic marking of coherence relations: A parallel corpus study. Journal of Pragmatics 121. 113-131. https://doi.org/10.1016/j.pragma.2017.10.010

Hoek, Jet, Sandrine Zufferey, Jacqueline Evers-Vermeul \& Ted J.M. Sanders. 2019. The linguistic marking of coherence relations: Interactions between connectives and segmentinternal elements. Pragmatics and Cognition 25(2). 275-309.

Kitis, Eliza. 2000. Connectives and frame theory: The case of hypotextual antinomial 'and'. Pragmatics and Cognition 8(2). 357-409. https://doi.org/10.1075/pc.8.2.04kit

Knott, Alistair \& Robert Dale. 1994. Using linguistic phenomena to motivate a set of coherence relations. Discourse Processes 18. 35-62. https://doi.org/10.1080/01638539409544883

Levy, Roger \& T. Florian Jaeger. 2007. Speakers optimize information density through syntactic reduction. In Bernhard Schölkopf, John Platt \& Thomas Hoffman (eds.), Advances in neural information processing systems (NIPS), 849-856. Cambridge, MA: MIT Press.

Luscher, Jean-Marc \& Jacques Moeschler. 1990. Approches dérivationnelles et procédurales des opérateurs et connecteurs temporels: Les exemples de et et de enfin. Cahiers de Linguistique Française 11. 77-104.

Mann, William C. \& Sandra A. Thompson. 1988. Rhetorical structure theory: Toward a functional theory of text organization. Text - Interdisciplinary Journal for the Study of Discourse 8. 243-281. https://doi.org/10.1515/text.1.1988.8.3.243

Millis, Keith K. \& Marcel A. Just. 1994. The influence of connectives on sentence comprehension. Journal of Memory and Language 33. 128-147. https://doi.org/10.1006/jmla.1994.1007

Murray, John. 1997. Connectives and narrative text: The role of continuity. Memory and Cognition 25(2). 227-236. https://doi.org/10.3758/BF03201114

Pander Maat, Henk. 1999. The differential linguistic realization of comparative and additive coherence relations. Cognitive Linguistics 10(2). 147-184.

Prasad, Rashmi, Nikhil Dinesh, Alan Lee, Eleni Miltsakaki, Livio Robaldo, Aravind Joshi \& Bonnie Webber. 2008. The Penn Discourse Treebank 2.o. Paper presented at the 6 th International Conference on Language Resources and Evaluation (LREC 2008), Marrakech, Morocco.

Prasad, Rashmi, Bonnie Webber \& Alan Lee. 2018. Discourse annotation in the PDTB: The next generation. In Harry Bunt (ed.), Proceedings of the 14th Joint ACL-ISO Workshop on Interoperable Semantic Annotation, 87-97. Santa Fe, NM: Association for Computational Linguistics.

Pusse, Florian, Sayeed Asad \& Vera Demberg. 2016. Lingoturk: Managing crowdsourced tasks for psycholinguistics. Proc. of the North American Chapter of the Association for Computational Linguistics. https://doi.org/10.18653/v1/N16-3012

Rohde, Hannah, Joseph Tyler \& Katy Carlson. 2017. Form and function: Optional complementizers reduce causal inferences. Glossa 2(1). https://doi.org/10.5334/gjgl.134

Sanders, Ted J.M. \& Leo Noordman. 200o. The role of coherence relations and their linguistic markers in text processing. Discourse Processes 29.37-60. https://doi.org/10.1207/S15326950dp2901_3

Sanders, Ted J.M., Wilbert Spooren \& Leo Noordman. 1993. Coherence relations in a cognitive theory of discourse representation. Cognitive Linguistics 4(2). 93-133.

https://doi.org/10.1515/cogl.1993.4.2.93 
Spooren, Wilbert. 1997. The processing of underspecified coherence relations. Discourse Processes 24. 149-168. https://doi.org/10.1080/01638539709545010

Traxler, Matthew, Michael Bybee \& Martin J. Pickering. 1997. Influence of connectives on language comprehension: Eye-tracking evidence for incremental interpretation. The Quarterly Journal of Experimental Psychology 50A(3). 481-497. https://doi.org/10.1080/027249897391982

Van Silfhout, Gerineke, Jacqueline Evers-Vermeul \& Ted J. M. Sanders. 2015. Connectives as processing signals: How students benefit in processing narrative and expository texts. Discourse Processes 52(1). 47-76. https://doi.org/10.1080/0163853X.2014.905237

Zufferey, Sandrine \& Liesbeth Degand. 2013. Annotating the meaning of discourse connectives in multilingual corpora. Corpus Linguistics and Linguistic Theory 13(2). 399-422. https://doi.org/10.1515/cllt-2013-0022

\title{
Address for correspondence
}

\author{
Ludivine Crible \\ Université catholique de Louvain \\ Place Blaise Pascal, 1 \\ 1348 Louvain-la-Neuve \\ Belgium \\ ludivine.crible@uclouvain.be
}

\section{Biographical notes}

Dr Ludivine Crible obtained her PhD in Linguistics at the Université catholique de Louvain in 2017, on the topic of discourse markers and disfluency in spoken English and French. She published several papers on corpus-based crosslinguistic studies of a range of phenomena including discourse markers and connectives. She is now integrating experimental methods in the investigation of the production, comprehension and processing of connectives in speech and writing.

Prof. Dr. Vera Demberg is a professor at the Department of Computer Science at Saarland University. She is also affiliated with the Department of Language Science and Technology. Vera obtained her $\mathrm{PhD}$ from the University of Edinburgh and has background in computational linguistics and psycholinguistics. A main focus of her research lies on discourse relation processing.

\section{Publication history}

Date accepted: 19 November 2020 